\title{
Extending the applicability of P3D for structure determination of small molecules
}

\author{
Alain Ibáñez de Opakua ${ }^{1}$ and Markus Zweckstetter ${ }^{1,2}$ \\ ${ }^{1}$ German Center for Neurodegenerative Diseases (DZNE), Von-Siebold-Str. 3a, 37075 Göttingen, Germany \\ ${ }^{2}$ Department for NMR-based Structural Biology, Max Planck Institute for Biophysical Chemistry, \\ Am Faßberg 11, 37077 Göttingen, Germany \\ Correspondence: Alain Ibáñez de Opakua (alain.ibanez-de-opakua@dzne.de) \\ and Markus Zweckstetter (markus.zweckstetter@dzne.de)
}

Received: 28 November 2020 - Discussion started: 7 December 2020

Revised: 15 March 2021 - Accepted: 15 March 2021 - Published: 8 April 2021

\begin{abstract}
The application of anisotropic nuclear magnetic resonance (NMR) parameters for the correct structural assignment of small molecules requires the use of partially ordered media. Previously we demonstrated that the use of P3D simulations using poly ( $\gamma$-benzyl-L-glutamate) (PBLG) as an alignment medium allows for the determination of the correct diastereomer from extremely sparse NMR data. Through the analysis of the structural characteristics of small molecules in different alignment media, here we show that when steric or electrostatic factors dominate the alignment, P3D-PBLG retains its diastereomer discrimination power. We also demonstrate that P3D simulations can define the different conformations of a flexible small molecule from sparse NMR data.
\end{abstract}

\section{Introduction}

Complete structure determination of small molecules, including stereochemistry, is a challenging task and an important step in organic chemistry and drug discovery. In recent years, anisotropic nuclear magnetic resonance (NMR) parameters, especially residual dipolar couplings (RDCs), have been used to determine the conformation of small organic molecules in organic solvents ( $\mathrm{Li}$ et al., 2018). RDCs provide a spatial view of the relative orientations of bonds, irrespective of internuclear distances being a good complement for conventional NMR restraints such as NOE (nuclear Overhauser effect) distances (Anet and Bourn, 1965) and dihedral angles from ${ }^{3} J$ spin-spin coupling constants (Haasnoot et al., 1980). However, RDCs cannot be measured in isotropic conditions because of the averaging of anisotropic NMR parameters by uniform molecular tumbling (Luy and Kessler, 2006). Thus, access to anisotropic NMR parameters requires the generation of anisotropic environments in solution, and this is achieved by using an alignment medium (Canet et al., 1995; Lesot et al., 1995, 1996a, 1996b; Tjandra and Bax, 1997).
There are two major types of alignment media with different inherent mechanisms to orient tumbling molecules: lyotropic liquid crystalline (LLC) phases and strain-induced alignment in a gel (SAG) (Tycko et al., 2000; Sass et al., 2000; Bax, 2003; Böttcher and Thiele, 2012; Canales et al., 2012; Leyendecker et al., 2017; Schmidts, 2017; Lesot et al., 2019; Krupp et al., 2021). The LLC phases spontaneously align in the presence of strong external magnetic fields because of their large magnetic susceptibility anisotropy. This alignment is then partially transmitted to the solvent and the molecules in solution with the degree and characteristics of the alignment depending on the concentration, temperature, and other parameters of the sample (Krupp and Reggelin, 2012). Alignment requires a minimum concentration of lyotropic medium and often aligns strongly at this concentration, which limits the tunability of the alignment strength. The degree of orientation in these media is therefore sometimes too large, complicating the extraction of RDCs. The strain-induced alignment in a gel (SAG) method generates the anisotropy mechanically, by compressing or stretching the gels. The alignment is then independent of the magnetic field and scalable over a wide range, making it easier to tune 
the alignment strength. However, sample preparation can be more difficult in the case of compressed and stretched gels. In addition, it often takes several days for the solute molecule to properly diffuse into the gel (Li et al., 2018).

Each alignment medium has its own degree of induced order and orientation of alignment. These properties can be influenced by experimental conditions and the properties of the small molecule to be aligned. How this happens is largely unknown. To fill this gap, we recently developed a three-dimensional molecular alignment model termed "P3D" (Ibáñez de Opakua et al., 2020). P3D allows for the establishment of a quantitative correlation between the atomic structure of the alignment medium, the molecular structure of the small molecule, and molecule-specific anisotropic NMR parameters. For the implementation of the model, we selected poly $(\gamma$-benzyl-L-glutamate) (PBLG) as the alignment medium. PBLG forms a LLC phase and has a well-defined $\alpha$-helical structure (Doty et al., 1954; Marx and Thiele, 2009). The P3D simulation uses a combination of steric obstruction and continuum electrostatics. Analysis of several small molecules demonstrated that the P3D model reliably discriminates between different relative configurations of small molecules dissolved in PBLG (Ibáñez de Opakua et al., 2020). To gain insight into the applicability of P3Dbased enantiomer discrimination when other alignment media are used, here we investigate how different the alignment of solutes dissolved in other alignment media is and how these differences are tuned by the type of solute. A better understanding of these questions will allow for the application of molecular alignment simulations performed for one alignment media to other media if the alignment is expected to be similar. In addition, an informed choice of the alignment medium might become possible when different alignments are required to discriminate between candidate structures (Ramirez and Bax, 1998).

The discrimination between different structures of a molecule is a major application of anisotropic NMR parameters (Zweckstetter and Bax, 2000). In the case of small molecules, the determination of the constitution by NMR spectroscopy is often a standard procedure, while the determination of its conformation and configuration can be more challenging. Relative configuration determination has been improved in the last years (Li et al., 2018; Liu et al., 2018; Ibáñez de Opakua et al., 2020), in particular through the use of RDCs and residual chemical shift anisotropies (RCSAs). In addition, a number of different chiral alignment media, which possess enantiodifferentiating properties, were developed (Marx et al., 2009; Arnold et al., 2010; Krupp and Reggelin, 2012; Meyer et al., 2012; Hansmann et al., 2016; Reller et al., 2017). However, it has so far not been possible to reliably predict the enantiodifferentiating properties from the molecular properties of the chiral alignment medium and the small molecule, leaving the determination of the absolute configuration on the basis of anisotropic NMR parameters an unsolved problem (Berger et al., 2012). In addition, flexi- ble small molecules with multiple conformations complicate the use of least-square methods to fit the experimental RDC and/or RCSA values to an ensemble of structures, especially if the conformers align with different alignment tensors. Here molecular alignment simulations might help to identify those structures that fit the experimental RDCs better. We therefore also investigate in the current study whether P3D simulations are not only beneficial for the analysis of the relative configuration of small molecules, but also for their conformational analysis.

\section{Methods}

Strychnine and isopinocampheol (IPC) structures were built as described previously (strychnine: Bifulco et al., 2013; IPC: Ibáñez de Opakua et al., 2020). RDCs of the molecules in the different alignment media were obtained from the references given in Table 1. Sucrose conformer structures and RDCs were obtained from Ndukwe et al. (2019).

Molecular alignment simulations (P3D), implemented in the software PALES (Zweckstetter, 2008), were performed as described previously (Ibáñez de Opakua et al., 2020). During the simulation, the solute molecule is moved in steps on a three-dimensional grid that covers the central part of PBLG. At each step, a uniform distribution of different solute molecule orientations is sampled. The spacing of the three-dimensional grid was set to $0.4 \AA$ and the number of sampled orientations to 1800 (100 orientations on the unit sphere and 18 in the third dimension). The interaction energy between the solute molecule and the PBLG particle is then calculated for each orientation and/or grid position on the basis of the precomputed potential file of the PBLG particle and the charges of the solute molecule. The interaction energy is converted into a Boltzmann weighing factor, and the RDCs are calculated. Charges of the small molecules were calculated using the AtomicChargeCalculator server (Ionescu et al., 2015) via the electronegativity equalization method based on a common charge calculation scheme (atoms in molecules) and a robust quantum mechanical approach (HF/6-311G). Therefore, the PALES command used to run the simulations is as follows:

pales -elPales -3D -pot3D PBLG.dx -lcS 0.8 -maxPot 2 -z1 150 -zN 250 -nX 129 -nY 129 -nZ 385 -dX 0.4 -dY 0.4 -dZ 0.4 -H -nosurf -pdb SmallMolecule.pdb -inD RDCs.tbl -wv 0.12 -rM 8 -pka charges.pka -outD output.out,

where PBLG.dx is the potential file of PBLG obtained from APBS, SmallMolecule.pdb is the PDB file of the small molecule, RDCs.tbl is the list of experimental RDCs, charges.pka is the list of atomic charges obtained from AtomicChargeCalculator server as explained, and output.out is the output file with the final results.

The alignment tensors for strychnine and (-)-IPC in the different alignment media and for the different conformers of 
Table 1. Selected alignment media for which experimental RDCs of strychnine and (-)-IPC were reported.

\begin{tabular}{|c|c|c|}
\hline \multicolumn{3}{|c|}{ Strychnine } \\
\hline PBLG & $\operatorname{poly}(\gamma$-benzyl-L-glutamate $)$ & Liu et al. (2018) \\
\hline PELG & $\operatorname{poly}(\gamma$-ethyl-L-glutamate $)$ & Thiele (2004) \\
\hline PMMA & poly(methyl methacrylate) & Nath et al. (2015) \\
\hline PIAF & poly(L-isocyanoalanyl-L-phenylalanine benzyl ester) & Li et al. (2017) \\
\hline PS & cross-linked polystyrene & Luy et al. (2004) \\
\hline PA1 & poly-A-1/L-alanine-derived polyacetylene & Dama and Berger (2012b) \\
\hline PL1 & poly-L-1/poly(phenylisocyanide) & Dama and Berger (2012a) \\
\hline PALV & L-valine-derived polyacetylene & Meyer et al. (2012) \\
\hline PADV & D-valine-derived polyacetylene & Meyer et al. (2012) \\
\hline \multicolumn{3}{|c|}{ (-)-IPC (isopinocampheol) } \\
\hline PBLG & $\operatorname{poly}(\gamma$-benzyl-L-glutamate $)$ & Marx et al. (2009) \\
\hline PBDG & $\operatorname{poly}(\gamma$-benzyl-D-glutamate $)$ & Marx et al. (2009) \\
\hline PELG & $\operatorname{poly}(\gamma$-ethyl-L-glutamate $)$ & Hansmann et al. (2016) \\
\hline PALF300 & L-phenylalanine-derived polyacetylene at $300 \mathrm{~K}$ & Krupp and Reggelin (2012) \\
\hline PALF316 & L-phenylalanine-derived polyacetylene at $316 \mathrm{~K}$ & Krupp and Reggelin (2012) \\
\hline PL1 & poly-L-1/poly(phenylisocyanide) & Reller et al. (2017) \\
\hline PALV & L-valine-derived polyacetylene & Meyer et al. (2012) \\
\hline PADV & D-valine-derived polyacetylene & Meyer et al. (2012) \\
\hline PPEMG & poly(N-methyl-N'-((R)-1-phenylethyl)guanidine) & Arnold et al. (2010) \\
\hline
\end{tabular}

sucrose were calculated by best-fitting experimental RDCs to the respective structures using singular value decomposition (SVD) as implemented in the software PALES (Zweckstetter, 2008). To evaluate the correlation between experimental and fitted/simulated RDCs, $R$ (Pearson's correlation coefficient) and RQ parameters are used. The quality parameter RQ is defined as $(R+1)^{2} / Q_{\mathrm{S}}$, where $Q_{\mathrm{S}}$ is the RDC quality factor $Q=\operatorname{rms}\left(\mathrm{D}^{\exp }-\mathrm{D}^{\mathrm{P} 3 \mathrm{D}}\right) / \mathrm{rms}\left(\mathrm{D}^{\mathrm{exp}}\right)$ scaled by the slope of the $\mathrm{D}^{\exp }$ vs. $\mathrm{D}^{\mathrm{P} 3 \mathrm{D}}$ fitting (Ibáñez de Opakua et al., 2020). Variations in the SVD-derived quality measures $R$ and RQ were evaluated using a Monte Carlo noise method (Zweckstetter and Bax, 2002), in which random noise was added to the experimental RDCs according to their estimated accuracy.

In order to calculate the relative populations of each sucrose conformer from the P3D-predicted RDCs, RQ values were maximized by a grid search over the conformer populations using steps of $1 \%$.

\section{Evaluation of the partial alignment of P3D-PBLG and different alignment media with two different solutes}

Several different alignment media for organic solvents are available nowadays. In order to evaluate the alignment properties of these media, we selected the two most widely studied molecules in the field: strychnine and isopinocampheol (IPC). Strychnine is a reference compound for relative configuration determination because of the high number of chiral centers (six chiral centers generating 13 diastereomers). In addition, the low flexibility of strychnine minimizes con- tributions from different conformations. IPC is also a rigid molecule, with little overlap in the two-dimensional protoncarbon correlation spectrum and with both enantiomers available. Because of these favorable properties, the groups of Reggelin and Thiele used IPC for the development of alignment media with enantiodifferentiating capabilities (Marx et al., 2009; Arnold et al., 2010; Krupp and Reggelin, 2012; Meyer et al., 2012; Hansmann et al., 2016; Reller et al., 2017) used IPC for the development of alignment media with enantiodifferentiating capabilities. As we are not focusing here on the absolute configuration problem, we selected the enantiomer (-)-IPC for the analysis. We further examined the literature and extracted a set of nine alignment media for each of the compounds from which five are shared. Table 1 shows a list of the 12 selected alignment media together with the references from where the data were taken. The structures of the basic units of the alignment media are displayed in Fig. 1.

From the 12 selected alignment media, 10 form LLC phases. PMMA and PS were used as compressed and stretched gels, respectively. The bias towards LLC phases likely arises because a major objective of the current development of new alignment media is enantiodifferentiation, for which helical chiral nonracemic polymers, capable of forming LLC phases, are good candidates. The LLCforming polymers include polyglutamates, polyisocyanates, polyacetylenes, polyisocyanides, and polyguanidines.

The experimental one-bond $\mathrm{CH}$ RDCs observed for strychnine and (-)-IPC in these alignment media were compared both among each other and with the RDCs calculated by the P3D simulation using the PBLG model (Figs. 2 and 

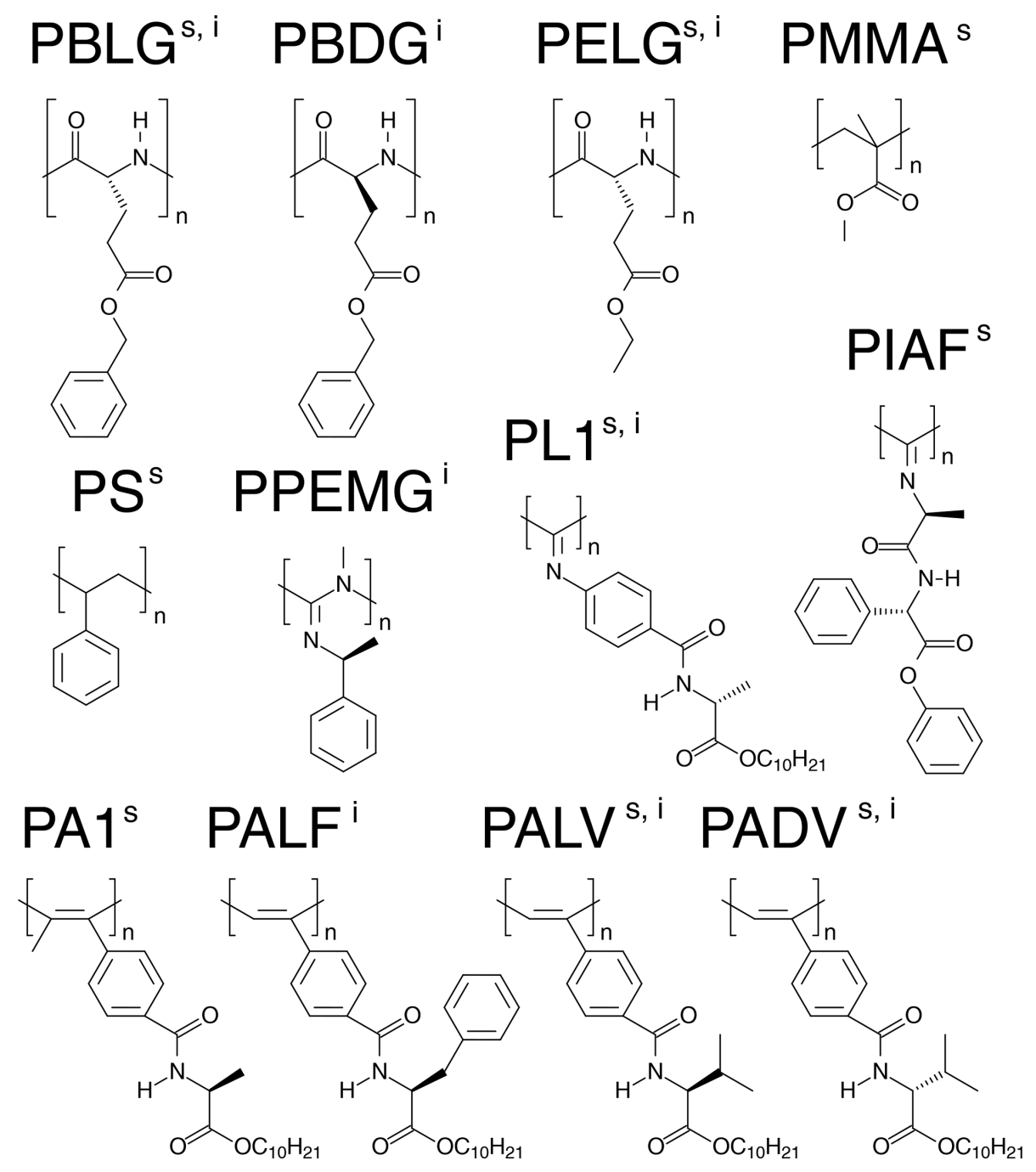

Figure 1. Representation of the basic units of the anisotropic media used for the alignment of strychnine (s) and (-)-IPC (i).

3). In the case of strychnine, half of the alignment media besides PBLG have a good correlation with the RDCs simulated by P3D on the basis of the PBLG alignment model (Fig. 3a). These alignment media are PELG, PMMA, PIAF, and PS. The experimental RDCs in the second group of alignment media (PA1, PL1, PALV, PADV) also largely correlate among each other but deviate more from the P3Dcalculated RDCs (Fig. 3a). This is confirmed by inspection of the alignment tensors (Fig. 3b): the orientation of the $z$ axis of the P3D-calculated alignment tensor is similar to that derived by singular value decomposition (SVD) from the experimental RDCs in many of the alignment media, with the exception of PA1 and PL1. Notably, a smaller number of RDCs were reported for PA1 and PL1 (five and six RDCs, respectively), which makes SVD-derived tensor orientations sensitive to the exact $\mathrm{CH}$ bond orientations in the structural models employed (Zweckstetter and Bax, 2002). We also point out that in some cases, alignment tensor axes were swapped; e.g., the $y$ axis is positioned where in other alignment media the $z$ axis is found (Fig. 3b). This can occur when two consecutive axes/eigenvalues have similar magnitude such that inaccuracies in experimental RDCs or molecular alignment simulation result in an exchange/relabeling of these axes, which however has only little influence on the backcalculated RDCs.

The alignment media that correlate better with the P3D prediction were further analyzed in Fig. 4. Comparison of P3D-predicted and experimental RDCs (Fig. 4a), as well as the alignment tensors projected onto a two-dimensional world map (Fig. 4c) and the aligned strychnine structures (Fig. 4d), shows that the partial ordering of strychnine in these alignment media is very similar and well predicted by P3D. Experimental RDCs observed in PMMA have a very good correlation with the P3D-calculated RDCs (Figs. 3a, 4a) and also a very similar alignment tensor (Fig. 4c), but the correlation is negative (Fig. 3a, dots). The negative slope in- 


\section{Strychnine}<smiles>[R7][R15][SeH]</smiles>

$\mathrm{RDCs}(\mathrm{Hz})$

\begin{tabular}{ccccccccccc} 
& P3D PBLG & PBLG & PELG & PMMA & PIAF & PS & PA1 & PL1 & PALV & PADV \\
\cline { 2 - 11 } $\mathrm{CH} 1$ & -114.9 & -178.9 & -43.5 & 37.0 & 19.6 & -9.3 & 8.5 & 35.0 & 27.9 & 19.9 \\
\hline $\mathrm{CH} 2$ & -118.1 & -36.9 & -15.3 & 22.8 & -3.3 & 0.8 & & -0.5 & -14.9 & -11.7 \\
\hline $\mathrm{CH} 3$ & -185.5 & -69.8 & -12.8 & 15.7 & 5.6 & & -16.6 & -18.4 & -11.8 & -5.2 \\
\hline $\mathrm{CH} 4$ & -115.7 & -182.4 & -43.5 & 37.8 & 21.2 & -10.5 & 10.6 & 39.5 & 28.0 & 21.5 \\
\hline $\mathrm{CH} 8$ & 84.5 & 87.1 & 24.6 & -17.5 & -11.0 & 3.6 & & -5.7 & & \\
\hline $\mathrm{CH} 12$ & 200.1 & 146.8 & 32.4 & -40.0 & -10.0 & 11.4 & & & -4.1 & -2.6 \\
\hline $\mathrm{CH} 13$ & 15.6 & 48.9 & 12.2 & -11.0 & -5.7 & -3.6 & 3.6 & & -5.8 & -4.8 \\
\hline $\mathrm{CH} 14$ & 184.1 & 151.7 & 31.5 & -38.7 & -11.5 & 12.0 & & & -8.4 & -5.4 \\
\hline $\mathrm{CH} 16$ & -101.1 & -49.6 & -14.8 & 25.1 & -1.9 & 1.8 & & -6.4 & & \\
\hline $\mathrm{CH} 22$ & -34.1 & -1.1 & 3.0 & -2.8 & 2.5 & -6.6 & 6.0 & & 1.0 & 0.0 \\
\hline
\end{tabular}

\section{(-)-IPC}

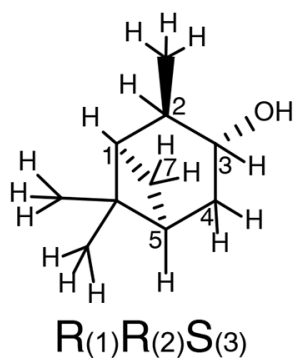

RDCs $(\mathrm{Hz})$

\begin{tabular}{|c|c|c|c|c|c|c|c|c|c|c|}
\hline & P3D PBLG & PBLG & PBDG & PELG & PALF300 & PALF316 & PL1 & $\overline{P A L V}$ & PADV & PPEMG \\
\hline $\mathrm{CH}_{1}{ }^{-}$ & 17.8 & 13.3 & 14.5 & 9.2 & 6.9 & 2.7 & 9.8 & -23.6 & 7.4 & 9.0 \\
\hline $\mathrm{CH} 2$ & 2.9 & -7.4 & -5.2 & -12.1 & 5.0 & 4.7 & 34.2 & 13.8 & 1.0 & 12.0 \\
\hline $\mathrm{CH} 3$ & 3.7 & 11.1 & 13.1 & 2.9 & 6.0 & -2.7 & 21.6 & 11.6 & 5.3 & 64.0 \\
\hline $\mathrm{CH} 5$ & 0.7 & -5.5 & -5.3 & -7.3 & -10.2 & 0.1 & -25.2 & -10.3 & -14.0 & 52.0 \\
\hline & 1.1 & -4.0 & -4.4 & -3.4 & -7.6 & -1.3 & -19.7 & 4.6 & -2.9 & -13.0 \\
\hline C & 10.8 & 10.9 & 14.3 & -2.4 & 19.0 & 5.5 & 56.2 & -3.8 & 10.0 & 40.0 \\
\hline & -27.5 & -16.4 & -17.4 & -9.8 & -4.8 & 2.2 & -0.5 & 15.1 & -1.9 & \\
\hline & -1.5 & 4.1 & 1.9 & 13.6 & 1.2 & -5.5 & -4.7 & 9.5 & 6.9 & \\
\hline
\end{tabular}

Figure 2. Strychnine and (-)-IPC structures together with the $\mathrm{CH}$ carbon labels and the correct configuration (left), as well as the respective lists of RDCs in the different alignment media (right).

dicates that the major alignment axis in PMMA is oriented orthogonal to the field, while PBLG aligns with its helix axis parallel to the magnetic field (Lorieau et al., 2008). Indeed, the PMMA gel was compressed, while the stretched PS gel displayed a positive correlation with the P3D-calculated RDCs. In other words, strychnine has in PMMA a highly similar alignment tensor to that in PBLG/PS but with an opposite sign of the axial component of the alignment $\left(\mathrm{D}_{\mathrm{a}}\right)$. For these reasons, when different alignment media are being compared, here we use the absolute value of the Pearson's correlation coefficient $R$.

We then investigated the diastereomer discrimination power of P3D-PBLG when using experimental RDCs observed in strychnine dissolved in the five different alignment media (Fig. 4b). To enhance the discrimination power, we use the quality parameter RQ. The ability of P3D-PBLG to select the correct diastereomer is retained for alignment of strychnine in PELG, PMMA, and PS. This is not the case for PIAF, which has a smaller Pearson's $R(0.71)$ value when compared to PELG, PMMA, and PS (all over 0.8) (Fig. 3a). This suggests that $R$ values larger than 0.8 are needed to identify the correct diastereomer, in agreement with the previously published P3D-based diastereomer discrimination analysis for six different small molecules (Ibáñez de Opakua et al., 2020).

Next, we performed the same P3D-based analysis for (-)IPC, which has different alignment properties when compared to strychnine. The correlation matrix for (-)-IPC only shows a strong correlation of P3D with PBLG and PBDG (Fig. 3c). In agreement with a weaker enantiodiscrimination power of PBLG when compared to PELG (Hansmann et al., 2016), or the other helical chiral nonracemic polymers shown here, the experimental RDCs observed for (-)-IPC in PBLG and PELG differ. On the other hand, PBLG and PBDG induce similar alignment such that the discrimination of different diastereomers of (-)-IPC was retained for PBDG. Notably, a change in the enantiomer of the alignment medium (e.g., from PBLG to PBDG) has the same effect as changing the enantiomer of the solute (e.g., from (-)-IPC to (+)-IPC) (Marx et al., 2009).

As far as correlations between experimental RDCs in different alignment media are concerned, only few media induce similar alignment of (-)-IPC (Fig. 3c). Only PL1, PALF300, and PADV form a small cluster in the correlation matrix. A correlation between experimental RDCs induced by PADV and PL1 was present for both (-)-IPC and strychnine, with $R$ values of 0.71 and 0.92 , respectively. The pronounced differences in the alignment of $(-)$-IPC in different alignment media are also evident from the comparison of the respective alignment tensors: the projected axes orientations do not cluster in certain regions (Fig. 3d), in contrast to the alignment tensors of strychnine (Fig. 3b). Further notable are the RDC differences when (-)-IPC is aligned in PALF300 and PALF316 $(R=0.45)$. This L-phenylalaninederived polyacetylene forms different LLC phases at differ- 

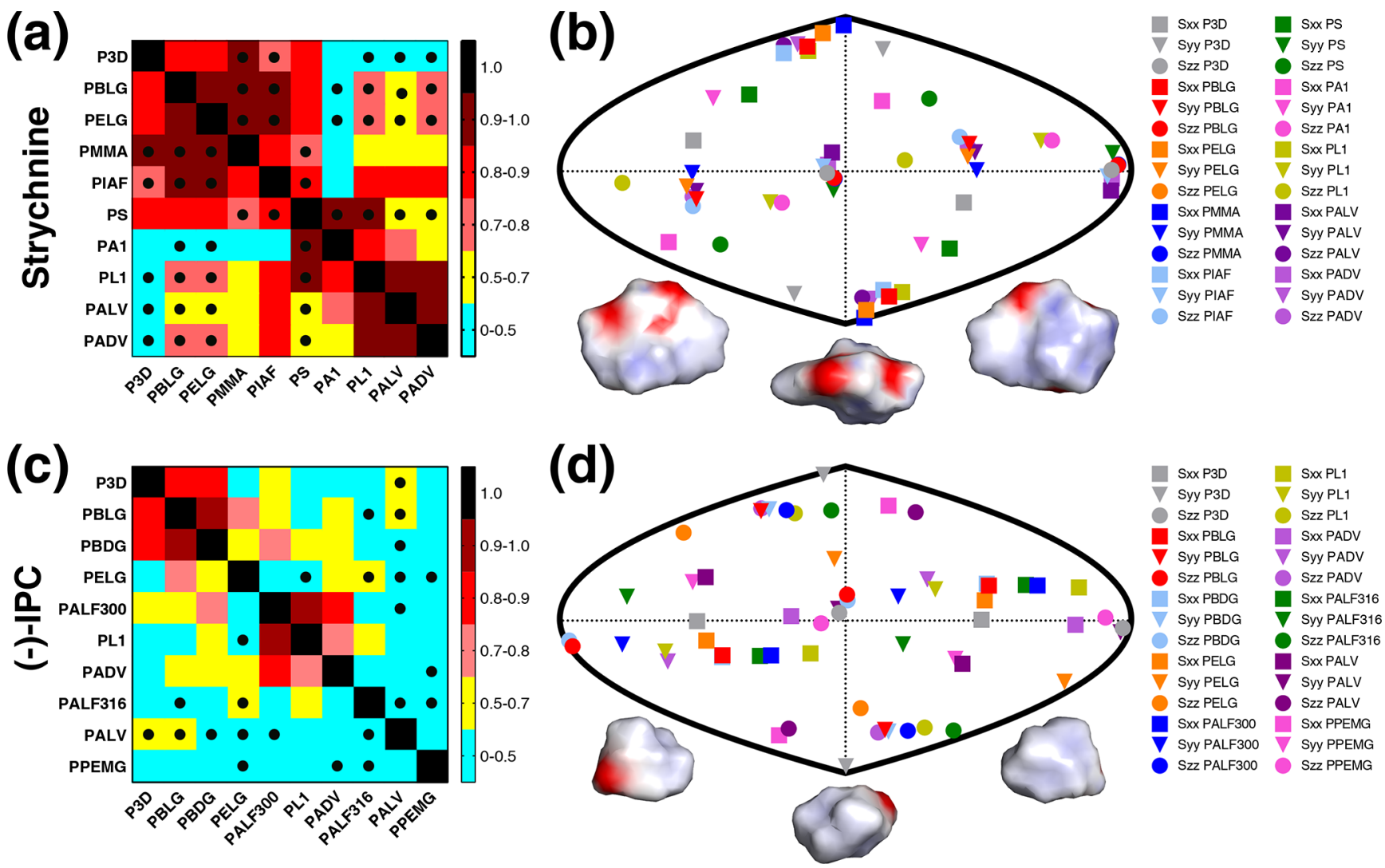

Figure 3. RDCs and alignment comparison of different alignment media and P3D for strychnine and (-)-IPC. (a, c) Matrices of Pearson's correlation $R$ between P3D-calculated RDCs and the experimental RDCs in different alignment media for strychnine (a) and (-)-IPC (c). Dots mark negative correlations. (b, d) Comparison of the orientation of the P3D-predicted alignment tensor (gray) with alignment tensors derived by SVD from experimental RDCs of the different alignment media for strychnine (b) and (-)-IPC (d). The orientations of the three axes corresponding to the eigenvalues Szz (circle), Syy (triangle), and Sxx (square) of the diagonalized alignment tensor are projected onto a two-dimensional world map. Different orientations of the charged surface of strychnine (b) and (-)-IPC (d) are shown below.

ent temperatures, with the helical structure severely disrupted at $316 \mathrm{~K}$ (Krupp and Reggelin, 2012). The pronounced differences in the alignment of (-)-IPC in PALF300 and PALF316 indicate that for certain molecules/alignment media, fine structural details of the alignment medium are critical for enantiodiscrimination.

In order to rationalize the distinct alignment properties of strychnine and IPC, we analyzed the structural properties of the two molecules (Fig. 3b, d). While strychnine has an oval disc-like shape, the shape of IPC is quite spherical, and both molecules have asymmetric charge distributions (Fig. 3b, d). Comparison of the correlation coefficients of the experimental RDCs with RDCs predicted by molecular alignment simulation using P3D or only steric interactions (1D obstruction model; Zweckstetter and Bax, 2000) suggested that electrostatic interactions are more important for the alignment of strychnine: $R$ values dropped from 0.88 to 0.64 in the case of strychnine and from 0.84 to 0.68 in the case of (-)-IPC, when replacing P3D simulations by 1D obstruction model simulations.
The excellent correlation between P3D-predicted and experimental RDCs of strychnine in PBLG indicates that the alignment of strychnine in PBLG is dominated by steric and electrostatic factors (Figs. 3, 4). At the same time, the fine structural details of the alignment media appear to be less important, which results in similar alignment of strychnine in PBLG, PELG, PMMA, and PS (Fig. 4). On the other hand, the quite spherical shape of IPC suggests that steric obstruction is less important for its molecular alignment. Instead, specific molecular interactions between IPC and the alignment medium become relevant and are responsible for the differences in RDC values observed in different alignment media. The difference in the alignment of IPC in PBLG and PELG might be correlated with the stronger enantiodifferentiating power of PELG, which has been linked to the change in the bulkiness and mobility of the lateral side chain (Hansmann et al., 2016). Due to these differences, IPC can have more and stronger diastereomorphous interactions with the chiral helical backbone of PELG. The importance of fine structural details for the alignment of IPC also provides a 

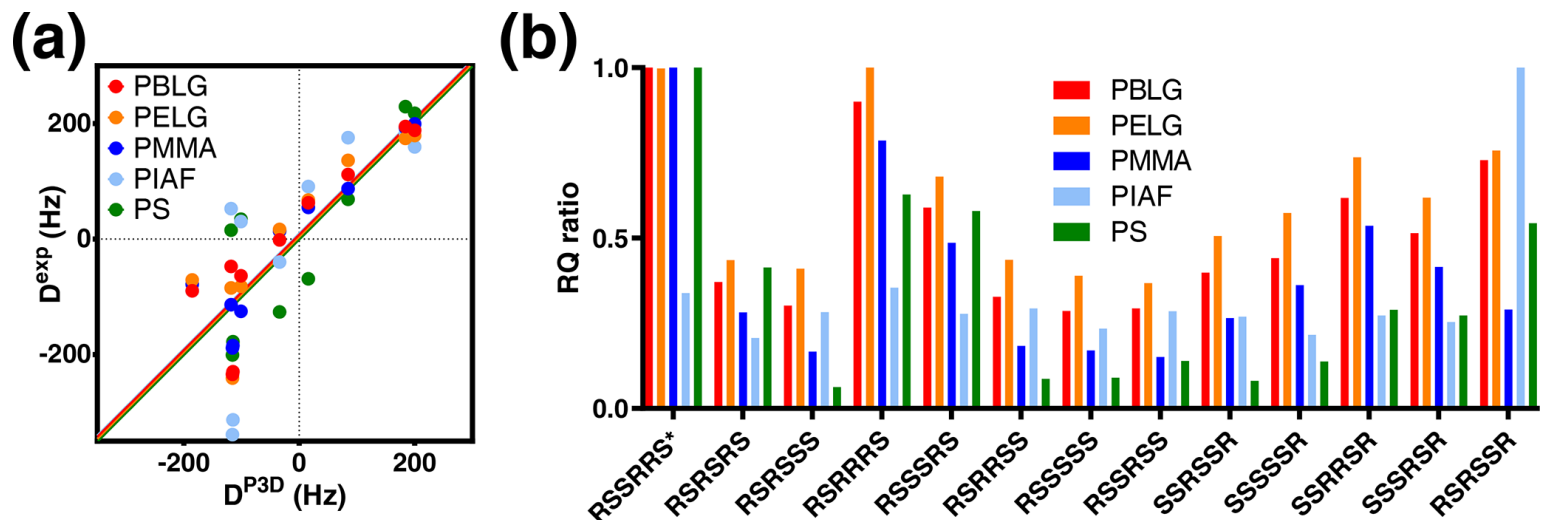

(c)
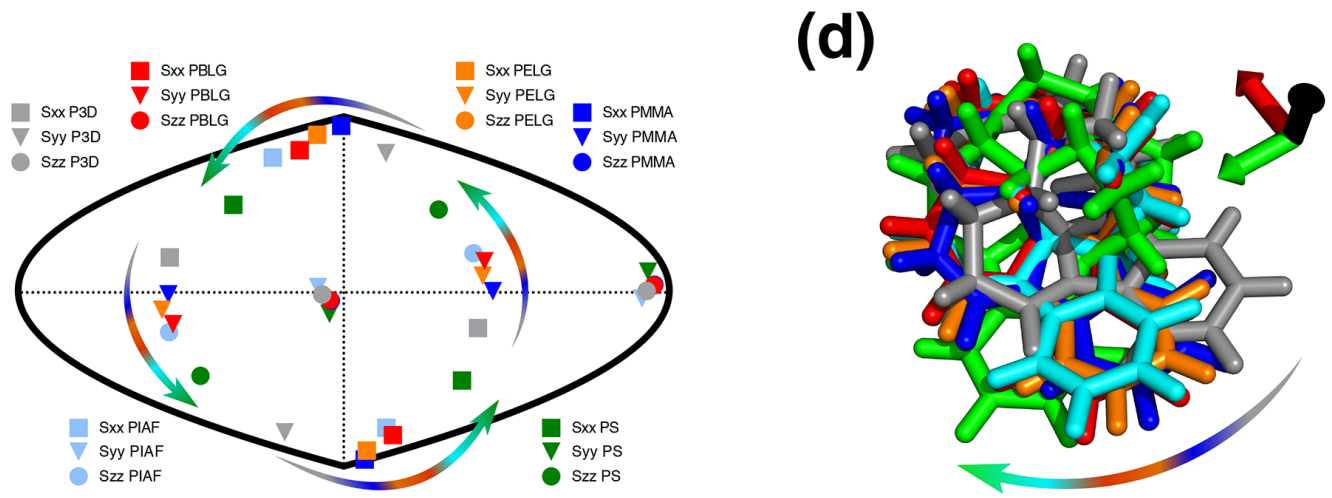

Figure 4. $\mathrm{P} 3 \mathrm{D}$ and the alignment media with most similar alignment properties for strychnine. (a) Correlation between P3D-simulated RDCs $\left(\mathrm{D}^{\mathrm{P} 3 \mathrm{D}}\right)$ and experimental RDCs $\left(\mathrm{D}^{\mathrm{exp}}\right)$ with different alignment media for strychnine. Experimental values are normalized by the slope of the linear fitting. (b) Diastereomer discrimination power of different alignment media for strychnine based on the P3D simulation and using the RQ ratio for judging the quality of correlation. (c) Comparison of the orientation of the P3D-predicted alignment tensor (gray) of strychnine with alignment tensors derived by SVD from the experimental RDCs using PALES (Zweckstetter, 2008) observed in different alignment media. The orientations of the three axes corresponding to the eigenvalues Szz (circle), Syy (triangle), and Sxx (square) of the diagonalized alignment tensor are projected onto a two-dimensional world map. (d) Oriented structures of strychnine according to the PBLG-based P3D simulation and the different experimentally analyzed alignment media. Axes colors are black $(z)$, red $(x)$, and green $(y)$ and are rotated when the axes of the alignment tensor are swapped because of a similar magnitude of the corresponding eigenvalues. The arrows in (c, d) illustrate the change in the orientation of the $x$ and $y$ axes in different alignment media.

rationale for why IPC is an excellent test molecule to study the enantiodifferentiation properties of alignment media.

\section{Analysis of conformational ensembles using P3D}

Because strychnine and IPC are rigid molecules, a single alignment tensor accurately describes their weak LLC/gelinduced alignment. However, for more flexible molecules, it is necessary to determine alignment tensors for all the conformers or independently for every flexible part of the molecule (Thiele and Berger, 2003). In order to simplify this problem, linearly independent alignment media would be needed (Ramirez and Bax, 1998), which, as shown in Fig. 3, is not always easy to achieve. When only one alignment medium is available, selection of energetically more promising structures and back-calculation of anisotropic NMR parameters to best-fit experimental values might be used (Tzvetkova et al., 2019). The latter approach, however, re- quires a large number of anisotropic NMR parameters and becomes difficult when the alignment tensors of the conformers are different.

We previously developed the P3D alignment simulation to solve the relative configuration problem, demonstrating that P3D can identify the correct diastereoisomer from a very small number of RDCs, even with fewer than five RDCs, the minimum number of RDCs required for SVD. Here we now use $\mathrm{P} 3 \mathrm{D}$ to address the problem of conformation. To this end, we selected sucrose (Fig. 5a), which has recently been analyzed by anisotropic NMR in PBLG (Ndukwe et al., 2019). Studies based on molecular dynamics (MD) simulations and solution NMR suggested the presence of multiple sucrose conformations (Venable et al., 2005; Xia and Case, 2012). On the basis of 11 RDCs and 12 RCSAs, the conformational ensemble of sucrose in $\mathrm{CDCl}_{3} / \mathrm{DMSO}(70: 30)$ was best described with three conformers, which were selected from a set of low-energy DFT (density functional theory) structures 


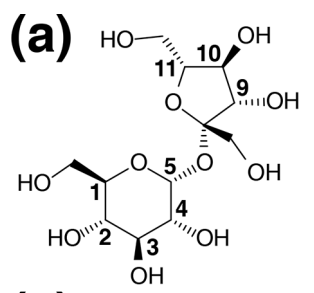

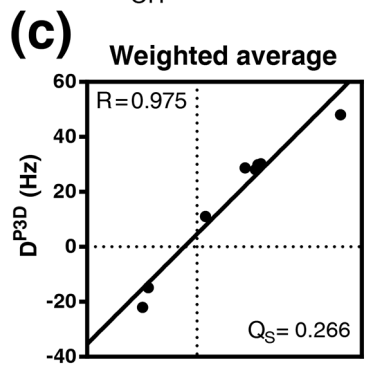

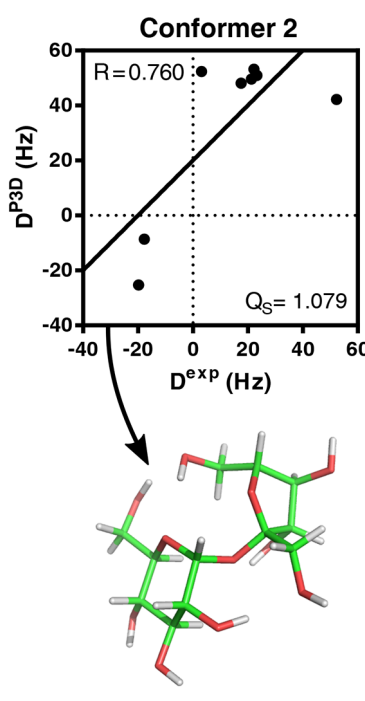

(b)

\begin{tabular}{lcccccccc} 
& \multicolumn{10}{c}{$\mathrm{RDCs}(\mathrm{Hz})$} \\
\cline { 2 - 9 } (b) & $\mathrm{CH} 1$ & $\mathrm{CH} 2$ & $\mathrm{CH} 3$ & $\mathrm{CH} 4$ & $\mathrm{CH} 5$ & $\mathrm{CH}$ & $\mathrm{CH} 10$ & $\mathrm{CH} 11$ \\
\cline { 2 - 9 } Conformer 1 (33\%) & 34.53 & 34.65 & 36.78 & 34.34 & 43.22 & -13.40 & -11.59 & -16.83 \\
\hline Conformer 2 (35\%) & 53.20 & 48.10 & 51.00 & 49.61 & 42.23 & -25.31 & 52.39 & -8.60 \\
\hline Conformer 3 (32\%) & -0.53 & 1.27 & 0.60 & -1.66 & 59.48 & -27.44 & -10.79 & -19.65 \\
\hline Weighted average & 29.85 & 28.68 & 30.18 & 28.17 & 48.08 & -22.06 & 11.06 & -14.85 \\
\hline Experimental & 22.23 & 17.53 & 23.32 & 21.3 & 52.25 & -19.79 & 3.12 & -17.74 \\
\hline
\end{tabular}

Conformer 1

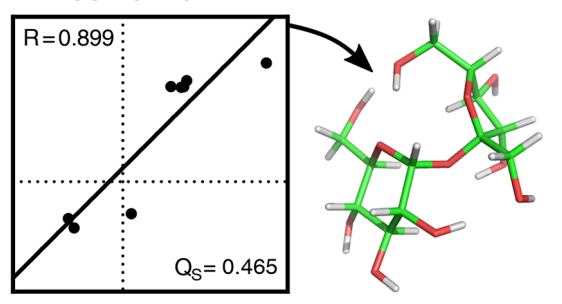

(d)

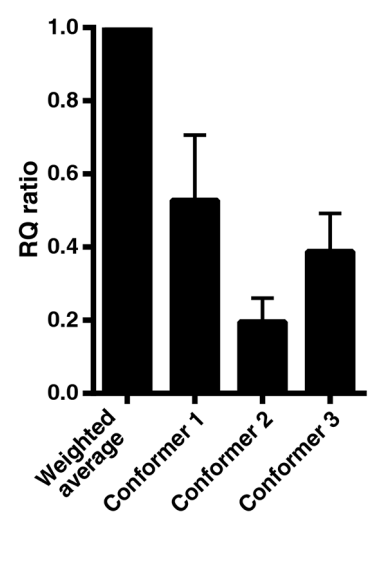

Figure 5. Validation of the conformational ensemble of sucrose using P3D. (a) Sucrose structure with the CH carbons labeled. (b) List of P3D-simulated RDCs for the three conformers together with the average weighted RDCs (from Ndukwe et al., 2019 populations) and the experimental RDCs. (c) Correlations between the experimental RDCs ( ${ }^{\mathrm{exp}}$ ) and the P3D-simulated RDCs for the three conformers and the weighted average. (d) RQ ratios of the three different conformers in reference to the weighted average. Error bars are calculated as the propagation of $R$ and $Q_{\mathrm{S}}$ errors, and these errors are calculated from the SD of 100 repetitions, including noise in the RDCs. (e) Comparison of the orientation of the P3D-predicted alignment tensors for the three conformers. The orientation of the three axes corresponding to the eigenvalues Szz (circle), Syy (triangle), and Sxx (square) of the diagonalized alignment tensor are projected onto a two-dimensional world map.

(Ndukwe et al., 2019). The respective $\Delta \mathrm{G}$ of conformers 1 , 2 , and 3 were $0,2.04$, and $2.72 \mathrm{kcal} \mathrm{mol}^{-1}$, with conformer 3 being highly similar to the crystal structure of sucrose (Russo et al., 2013).

Instead of the 23 anisotropic NMR parameters used by Ndukwe and colleagues (Ndukwe et al., 2019), here we use only the eight one-bond CH RDCs (Fig. 5a, b). Following the same rationale as before (Ibáñez de Opakua et al., 2020), we selected the one-bond CH RDCs (Fig. 5b) because they are the largest RDCs in small molecules; i.e., they can be measured with high accuracy, and there is less ambiguity in the assignment. We then subjected the three conformers of sucrose to P3D alignment simulation. P3D-simulated RDCs were averaged over the three-member ensemble and compared with the experimental RDCs (Fig. 5b, c). The results indicate that the three-member ensemble of conformers improved the correlation, reaching a $R$ value of 0.975 (Fig. 5c). The RQ of the weighted average is also significantly larger than the RQ values for any of the three individual conformers (Fig. 5d).

The structures of the three conformers were aligned before the simulation in order that they have the same molecu- 
(a)

\begin{tabular}{lcccccccc} 
& \multicolumn{10}{c}{$\mathrm{RDCs}(\mathrm{Hz})$} \\
\cline { 2 - 9 } Conformer 1 & $\mathrm{CH} 1$ & $\mathrm{CH} 2$ & $\mathrm{CH} 3$ & $\mathrm{CH} 4$ & $\mathrm{CH} 5$ & $\mathrm{CH} 9$ & $\mathrm{CH} 10$ & $\mathrm{CH} 11$ \\
\cline { 2 - 9 } & 34.53 & 34.65 & 36.78 & 34.34 & 43.22 & -13.40 & -11.59 & -16.83 \\
\hline Conformer 2 & 53.20 & 48.10 & 51.00 & 49.61 & 42.23 & -25.31 & 52.39 & -8.60 \\
\hline Conformer 3 & -0.53 & 1.27 & 0.60 & -1.66 & 59.48 & -27.44 & -10.79 & -19.65 \\
\hline Conformers 1/2 (w. a. 97/3\%) & 35.09 & 35.05 & 37.21 & 34.80 & 43.19 & -13.76 & -9.67 & -16.58 \\
\hline Conformers 1/3 (w. a. 59/41\%) & 20.16 & 20.96 & 21.95 & 19.58 & 49.89 & -19.16 & -11.26 & -18.00 \\
\hline Conformers 2/3 (w. a. 36/64\%) & 18.81 & 18.13 & 18.74 & 16.80 & 53.27 & -26.67 & 11.95 & -15.67 \\
\hline Conformers 1/2/3 (w. a. 29/22/49\%) & 21.46 & 21.25 & 22.18 & 20.06 & 50.97 & -22.90 & 2.88 & -16.40 \\
\hline Experimental & 22.23 & 17.53 & 23.32 & 21.3 & 52.25 & -19.79 & 3.12 & -17.74 \\
\hline
\end{tabular}

(c)

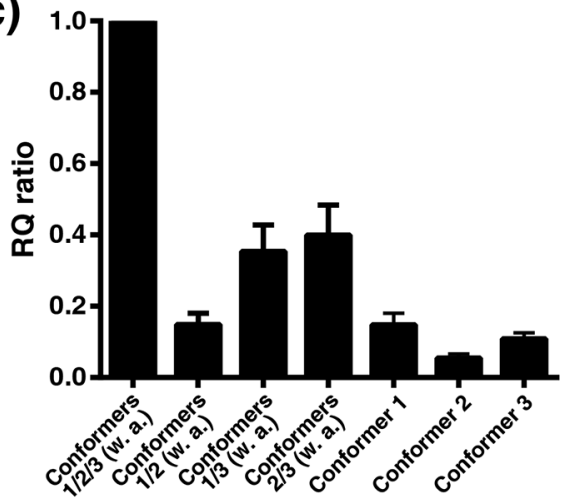

(b) Conformers $1 / 2$

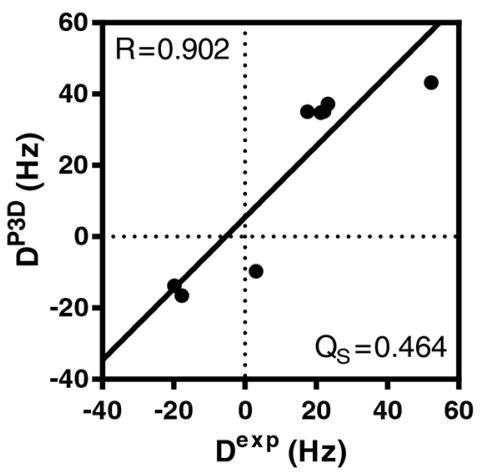

Conformers $1 / 3$

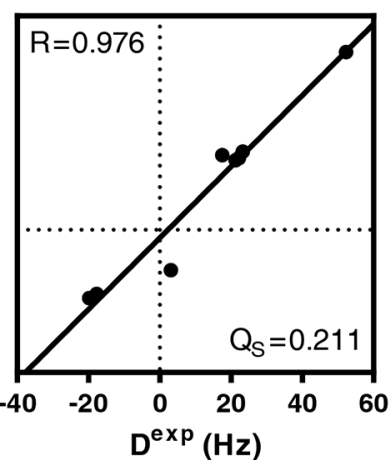

Conformers $2 / 3$

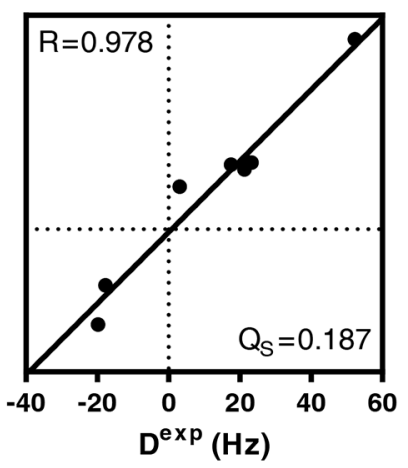

Conformers $1 / 2 / 3$

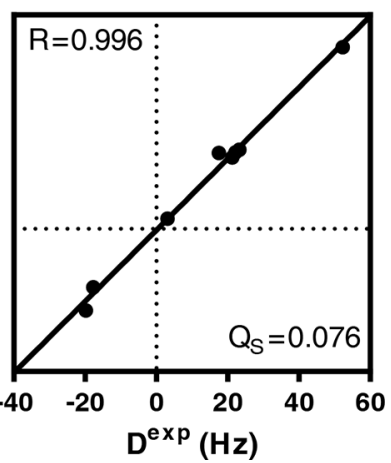

Figure 6. P3D-based refinement of the conformational ensemble of sucrose. (a) List of P3D-calculated RDCs for the three conformers together with the average weighted RDCs from the RQ maximization for two- and three-conformer ensembles and the experimental RDCs. (b) Correlation between the experimental RDCs. When all RDCs are considered, we get $R=0.926$ for the average with $0.806,0.615$, and 0.838 respectively for each conformer. The obtained populations $(26 \% / 20 \% / 54 \%)$ are very similar to the ones obtained with the one-bond CH RDCs only. ( $\mathrm{D}^{\exp }$ ) and the P3D-calculated RDCs for the weighted average of the RQ maximization for two- and three-conformer ensembles. (c) RQ ratios of the three different conformers in reference to their weighted average as two- and three-conformer ensembles. Error bars were calculated as the propagation of $R$ and QS errors, and these errors are calculated from the SD of 100 repetitions including noise in the RDCs.

lar frame and to enable comparison of the alignment tensors (Fig. 5e). The result shows that all the conformers have a similar but not identical alignment, indicating that the differences in RDCs come mainly from the structural differences, in agreement with the use of the variable-weight singletensor SVD method to solve the conformational structure of the molecule (Ndukwe et al., 2019).

While the variable-weight single-tensor SVD method strongly relies on the assumption that different conformers have similar alignment tensors, this is not required for the P3D-based conformational analysis. We therefore determined the relative numbers of conformers by maximizing the P3D-based RQ parameter (Fig. 6a, b). The comparison of the $\mathrm{RQ}$ ratios of the RQ-maximized three-conformer ensemble and the RQ-maximized two-conformer ensembles (Fig. 6c) shows that three is the minimum number of conformers to get an almost perfect fit $(R=0.996$; $\mathrm{QS}=0.076)$. In addition, the contribution of conformer 3 was increased to $49 \%$ (32\% in Ndukwe et al., 2019) in the refined three-conformer ensemble (Fig. 6a, b). Notably, the most populated conformer (conformer 3 ) is closest to the crystal structure of sucrose.

\section{Conclusions}

The current study highlights the important role of molecular alignment simulations in the structural analysis of small molecules. In agreement with previous data (Ibáñez de Opakua et al., 2020), the new analysis supports the applicability of P3D simulations for the determination of the relative configurations but also extends it to the analysis of conformational ensembles of small molecules. In addition, molecular alignment simulations might - with further improvements become crucial for the determination of the absolute configuration. While much progress has been made in the development of powerful chiral alignment media, this is restricted to the differentiation between enantiomers, similar to exposing small molecules to polarized light. To determine the absolute configuration, atomistic descriptions are required that 
link the NMR anisotropic parameters obtained from chiral alignment media with the correct enantiomer. A next step towards this goal could be the inclusion of specific interactions between the solute and the alignment medium, for example salt bridges, into molecular alignment simulations and the consideration of thermodynamic and kinetic contributions.

Towards this next step, it is important to define an alignment medium, which has a structure amenable to structural modeling and strong enantiodiscrimination capabilities. We therefore analyzed different alignment media and compared them with our P3D alignment simulation model of PBLG. A literature search identified only two molecules, strychnine and IPC, for which RDCs in several different alignment media had been reported. The results of our analysis suggested that the weak alignment induced by LLC phases and gels critically depends on both the molecular properties of the alignment medium and the small molecule. The comparison further showed that the alignment of IPC varies more strongly across the available alignment media when compared to strychnine (Fig. 3). We interpret this as a consequence of the more symmetrical/spherical shape of IPC such that more specific interactions with the alignment medium more strongly contribute to the alignment process. In the case of strychnine, on the other hand, steric obstruction together with electrostatic interactions dominate molecular ordering. This leads to less variability in the partial alignment of the small molecule, allowing for the application of the P3DPBLG model to other alignment media (Fig. 4). The choice of alignment medium should therefore take into account the structural properties of the small molecule of interest, especially its shape as well as the charge distribution.

We also investigated the applicability of the P3D-PBLG simulation approach to the challenge of determining conformational ensembles of flexible small molecules. With the example of sucrose, we showed that P3D can be used to determine the populations of different conformers in an ensemble, with the advantage that it can work even when individual conformers have different alignment tensors. Through the P3D-based analysis, we optimized the population of each conformer in the ensemble. The analysis resulted in an ensemble in which the population of conformer 3, which is closest to the crystal structure of sucrose, was increased to almost $50 \%$ (Fig. 6). The lower population of conformer 3 in the SVD-based ensemble might arise from the slightly different alignment tensors of the three conformers (Fig. 5e).

In summary, P3D alignment simulations establish a quantitative connection between the alignment medium, the molecular structure of small molecules, and anisotropybased NMR parameters. P3D can therefore predict RDCs for different alignment media depending on the structural details of both the alignment medium and the small molecule. As tested with the example of sucrose, P3D is also a promising approach and a preliminary but potentially feasible method for the determination of conformational ensembles of flexible small molecules.
Data availability. The Linux version of the PALES software with the P3D algorithm used in this work can be downloaded from the PALES web page (https://www3.mpibpc.mpg.de/groups/ zweckstetter/_links/software_pales.htm, last access: 24 March 2021, Klama and Zweckstetter, 2021). All other data that support the findings of this study are available from the corresponding authors upon reasonable request.

Author contributions. AIdO and $\mathrm{MZ}$ designed the project and wrote the paper. AIdO conducted the data analysis.

Competing interests. The authors declare that they have no conflict of interest.

Special issue statement. This article is part of the special issue "Robert Kaptein Festschrift". It is not associated with a conference.

Acknowledgements. Markus Zweckstetter acknowledges the financial support from the Helmholtz Society through the Impulsund Vernetzungsfond "Multiscale Bioimaging: From Molecular Machines to Networks of Excitable Cells" and from the European Research Council.

Financial support. This research has been supported by the European Research Council, H2020 European Research Council (LLPS-NMR; grant no. 787679).

The article processing charges for this open-access publication were covered by the Max Planck Society.

Review statement. This paper was edited by Rolf Boelens and reviewed by five anonymous referees.

\section{References}

Anet, F. A. L. and Bourn, A. J. R.: Nuclear magnetic resonance spectral assignments from nuclear Overhauser effects, J. Am. Chem. Soc., 87, 5250-5251, https://doi.org/10.1021/ja00950a048, 1965.

Arnold, L., Marx, A., Thiele, C. M., and Reggelin, M.: Polyguanidines as chiral orienting media for organic compounds, Chem.-Eur. J., 16, 10342-10346, https://doi.org/10.1002/chem.201000940, 2010.

Bax, A.: Weak alignment offers new NMR opportunities to study protein structure and dynamics, Protein Sci., 12, 1-16, https://doi.org/10.1110/ps.0233303, 2003.

Berger, R., Courtieu, J., Gil, R. R., Griesinger, C., Köck, M., Lesot, P., Luy, B., Merlet, D., Navarro-Vázquez, A., Reggelin, M., Reinscheid, U. M., Thiele, C. M., and Zweckstetter, M.: Is enantiomer assignment possible by NMR spectroscopy using residual dipolar couplings from chiral nonracemic alignment media? 
- A critical assessment, Angew. Chem. Int. Edit., 51, 8388-8391, https://doi.org/10.1002/anie.201107626, 2012.

Bifulco, G., Riccio, R., Martin, G. E., Buevich, A. V., and Williamson, R. T.: Quantum chemical calculations of $1 \mathrm{~J}(\mathrm{CC})$ coupling constants for the stereochemical determination of organic compounds, Org. Lett., 15, 654-657, https://doi.org/10.1021/ol3034803, 2013.

Böttcher, B. and Thiele, C. M.: Determining the Stereochemistry of Molecules from Residual Dipolar Couplings (RDCs), eMagRes, 1, 169-180, https://doi.org/10.1002/9780470034590.emrstm1194, 2012.

Canales, A., Jiménez-Barbero, J., and Martín-Pastor, M.: Review: Use of residual dipolar couplings to determine the structure of carbohydrates, Magn. Reson. Chem., 50, 80-85, https://doi.org/10.1002/mrc.3888, 2012.

Canet, I., Courtieu, J., Loewenstein, A., Meddour, A., and Pechine, J. M.: Enantiomeric analysis in a polypeptide lyotropic liquid crystal by deuterium NMR, J. Am. Chem. Soc., 117, 6520-6526, https://doi.org/10.1021/ja00129a015, 1995.

Dama, M. and Berger, S.: Polyisocyanides as a new alignment medium to measure residual dipolar couplings for small organic molecules, Org. Lett., 14, 241-243, https://doi.org/10.1021/ol202547y, 2012a.

Dama, M. and Berger, S.: Polyacetylenes as a new alignment medium to measure residual dipolar couplings for chiral organic molecules, Tetrahedron Lett., 53, 6439-6442, https://doi.org/10.1016/j.tetlet.2012.09.060, 2012b.

Doty, P., Holtzer, A. M., Bradbury, J. H., and Blout, E. R.: Polypeptides, II. The Configuration of Polymers of Gamma-BenzylL-Glutamate in Solution, J. Am. Chem. Soc., 76, 4493-4494, https://doi.org/10.1021/ja01646a079, 1954.

Haasnoot, C. A. G., de Leeuw, F. A. A. M., and Altona, C.: The relationship between proton-proton NMR coupling constants and substituent electronegativities - I: An empirical generalization of the karplus equation, Tetrahedron, 36, 2783-2792, https://doi.org/10.1016/0040-4020(80)80155-4, 1980.

Hansmann, S., Larem, T., and Thiele, C. M.: Enantiodifferentiating Properties of the Alignment Media PELG and PBLG - A Comparison, Eur. J. Org. Chem., 7, 1324-1329, https://doi.org/10.1002/ejoc.201501410, 2016.

Ibáñez de Opakua, A., Klama, F., Ndukwe, I. E., Martin, G. E., Williamson, R. T., and Zweckstetter, M.: Determination of Complex Small-Molecule Structures Using Molecular Alignment Simulation, Angew. Chem. Int. Edit., 59, 6172-6176, https://doi.org/10.1002/anie.202000311, 2020.

Ionescu, C. M., Sehnal, D., Falginella, F. L., Pant, P., Pravda, L., Bouchal, T., Vařeková, R. S., Geidl, S., and Koča, J.: Atomic Charge Calculator: interactive web-based calculation of atomic charges in large biomolecular complexes and drug-like molecules, J. Cheminformatics, 7, 50, https://doi.org/10.1186/s13321-015-0099-x, 2015.

Klama, F. and Zweckstetter, M.: PALES software, available at: https://www3.mpibpc.mpg.de/groups/zweckstetter/_links/ software_pales.htm, last access: 24 March 2021.

Krupp, A. and Reggelin, M.: Phenylalanine-based polyarylacetylenes as enantiomer-differentiating alignment media, Magn. Reson. Chem., 50, 45-52, https://doi.org/10.1002/mrc.3894, 2012.
Krupp, A., Noll, M., and Reggelin, M.: Valine derived poly (acetylenes) as versatile chiral lyotropic liquid crystalline alignment media for RDC-based structure elucidations, Magn. Reson. Chem., 1-10, https://doi.org/10.1002/mrc.5003, online first, 2021.

Lesot, P., Merlet, D., Meddour, A., Courtieu, J., and Loewenstein, A.: Visualization of Enantiomers in a Polypeptide Liquid-Crystal Solvent through carbon-13 NMR Spectroscopy, J. Chem. Soc. Faraday T., 91, 1371-1375, https://doi.org/10.1039/ft9959101371, 1995.

Lesot, P., Merlet, D., Courtieu, J., and Emsley, J. W.: Discrimination and analysis of the NMR spectra of enantiomers dissolved in chiral liquid crystal solvents through 2D correlation experiments, Liq. Cryst., 21, 427-435, https://doi.org/10.1080/02678299608032851, 1996a.

Lesot, P., Gounelle, Y., Merlet, D., Loewenstein, A., and Courtieu, J.: Measurement and Analysis of the Molecular Ordering Tensors of 2 Enantiomers Oriented in a Polypeptide LiquidCrystalline System, J. Phys. Chem.-US, 99, 14871-14875, https://doi.org/10.1021/jp961819v, 1996b.

Lesot, P., Berdague, P., Meddour, A., Kreiter, A., Noll, M., and Reggelin, M.: ${ }^{2} \mathrm{H}$ and ${ }^{13} \mathrm{C}$ NMR-Based Enantiodetection Using Polyacetylene versus Polypeptide Aligning Media: Versatile and Complementary Tools for Chemists, Chempluschem, 84, 144153, https://doi.org/10.1002/cplu.201800493, 2019.

Leyendecker, M., Meyer, N. C., and Thiele, C. M.: Development of New Supramolecular Lyotropic Liquid Crystals and Their Application as Alignment Media for Organic Compounds, Angew. Chem. Int. Edit., 56, 11471-11474, https://doi.org/10.1002/anie.201705642, 2017.

Li, G. W., Cao, J. M., Zong, W., Hu, L., Hu, M. L., Lei, X., Sun, H., and Tan, R. X.: Helical Polyisocyanopeptides as Lyotropic Liquid Crystals for Measuring Residual Dipolar Couplings, Chem.-Eur. J., 23, 7653-7656, https://doi.org/10.1002/chem.201700539, 2017.

Li, G. W., Liu, H., Qiu, F., Wang, X. J., and Lei, X. X.: Residual Dipolar Couplings in Structure Determination of Natural Products, Natural Products and Bioprospecting, 8, 279-295, https://doi.org/10.1007/s13659-018-0174-x, 2018.

Liu, Y., Cohen, R. D., Martin, G. E., and Williamson, R. T.: A practical strategy for the accurate measurement of residual dipolar couplings in strongly aligned small molecules, J. Magn. Reson., 291, 63-72, https://doi.org/10.1016/j.jmr.2018.04.002, 2018.

Lorieau, J., Yao, L., and Bax, A.: Liquid Crystalline Phase of G-Tetrad DNA for NMR Study of DetergentSolubilized Proteins, J. Am. Chem. Soc., 130, 7536-7537, https://doi.org/10.1021/ja801729f, 2008.

Luy, B. and Kessler, H.: Partial Alignment for Structure Determination of Organic Molecules, in: Modern Magnetic Resonance, Springer International Publishing, Dordrecht, The Netherlands, 1279-1285, https://doi.org/10.1007/1-4020-3910-7, 2006.

Luy, B., Kobzar, K., and Kessler, H.: An easy and scalable method for the partial alignment of organic molecules for measuring residual dipolar couplings, Angew. Chem. Int. Edit., 43, 10921094, https://doi.org/10.1002/anie.200352860, 2004.

Marx, A. and Thiele, C. M.: Orientational properties of PBLG: Influence of molecular weight and solvent on order parameters of the solute, Chem.-Eur. J., 15, 254-260, https://doi.org/10.1002/chem.200801147, 2009. 
Marx, A., Schmidts, V., and Thiele, C. M.: How different are diastereomorphous orientations of enantiomers in the liquid crystalline phases of PBLG and PBDG: a case study, Magn. Reson. Chem., 47, 734-740, https://doi.org/10.1002/mrc.2454, 2009.

Meyer, N. C., Krupp, A., Schmidts, V., Thiele, C. M., and Reggelin, M.: Polyacetylenes as enantiodifferentiating alignment media, Angew. Chem. Int. Edit., 51, 8334-8338, https://doi.org/10.1002/anie.201201891, 2012.

Nath, N., d'Auvergne, E. J., and Griesinger, C.: Long-Range Residual Dipolar Couplings: A Tool for Determining the Configuration of Small Molecules, Angew. Chem. Int. Edit., 54, 12706-12710, https://doi.org/10.1002/anie.201504432, 2015.

Ndukwe, I. E., Wang, X., Pelczer, I., Reibarkh, M., Williamson, R. T., Liu, Y., and Martin, G. E.: PBLG as a Versatile Liquid Crystalline Medium for Anisotropic NMR Data Acquisition, Chem. Commun., 55, 4327-4330, https://doi.org/10.1039/C9CC01130G, 2019.

Ramirez, B. E. and Bax, A.: Modulation of the Alignment Tensor of Macromolecules Dissolved in a Dilute Liquid Crystalline Medium, J. Am. Chem. Soc., 120, 9106-9107, https://doi.org/10.1021/ja982310b, 1998.

Reller, M., Wesp, S., Koos, M. R. M., Reggelin, M., and Luy B.: Biphasic Liquid Crystal and the Simultaneous Measurement of Isotropic and Anisotropic Parameters by Spatially Resolved NMR Spectroscopy, Chem.-Eur. J., 23, 13351-13359, https://doi.org/10.1002/chem.201702126, 2017.

Russo, L., Grazulis, S., and Bagdziunas, G.: Structure of Sucrose, Personal communication to Crystallography Open Database (COD), available at: http://www.crystallography.net/ cod/3500015.html (last access: 24 March 2021), 2013.

Sass, H. J., Musco, G., Stahl, S. J., Wingfield, P. T., and Grzesiek, S.: Solution NMR of proteins within polyacrylamide gels: Diffusional properties and residual alignment by mechanical stress or embedding of oriented purple membranes, J. Biomol. NMR, 18, 303-309, https://doi.org/10.1023/A:1026703605147, 2000.

Schmidts, V.: Perspectives in the application of residual dipolar couplings in the structure elucidation of weakly aligned small molecules, Magn. Reson. Chem., 55, 54-60, https://doi.org/10.1002/mrc.4543, 2017.
Thiele, C. M.: Simultaneous assignment of all diastereotopic protons in strychnine using RDCs: PELG as alignment medium for organic molecules, J. Org. Chem., 69, 7403-7413, https://doi.org/10.1021/jo049867w, 2004.

Thiele, C. M. and Berger, S.: Probing the Diastereotopicity of Methylene Protons in Strychnine Using Residual Dipolar Couplings, Org. Lett., 5, 705-708, https://doi.org/10.1021/o10275163, 2003.

Tjandra, N. and Bax, A.: Direct measurement of distances and angles in biomolecules by NMR in a dilute liquid crystalline medium, Science, 278, 1111-1114, https://doi.org/10.1126/science.278.5340.1111, 1997.

Tycko, R., Blanco, F. J., and Ishii, Y.: Alignment of Biopolymers in Strained Gels: A New Way To Create Detectable Dipole-Dipole Couplings in High-Resolution Biomolecular NMR, J. Am. Chem. Soc., 122, 9340-9341, https://doi.org/10.1021/ja002133q, 2000.

Tzvetkova, P., Sternberg, U., Gloge, T., Navarro-Vázquez, A., and Luy, B.: Configuration determination by residual dipolar couplings: accessing the full conformational space by molecular dynamics with tensorial constraints, Chem. Sci., 10, 8774-8791, https://doi.org/10.1039/C9SC01084J, 2019.

Venable, R. M., Delaglio, F., Norris, S. E., and Freedberg, D. I.: The utility of residual dipolar couplings in detecting motion in carbohydrates: application to sucrose, Carbohyd. Res., 340, 863874, https://doi.org/10.1016/j.carres.2005.01.025, 2005.

Xia, J. and Case, D. A.: Sucrose in aqueous solution revisited, Biopolymers, 97, 276-288, https://doi.org/10.1002/bip.22017, 2012.

Zweckstetter, M.: NMR: prediction of molecular alignment from structure using the PALES software, Nat. Protoc., 3, 679-690, https://doi.org/10.1038/nprot.2008.36, 2008.

Zweckstetter, M. and Bax, A.: Prediction of Sterically Induced Alignment in a Dilute Liquid Crystalline Phase: Aid to Protein Structure Determination by NMR, J. Am. Chem. Soc., 122, 3791-3792, https://doi.org/10.1021/ja0000908, 2000.

Zweckstetter, M. and Bax, A.: Evaluation of uncertainty in alignment tensors obtained from dipolar couplings, J. Biomol. NMR, 23, 127-137, https://doi.org/10.1023/A:1016316415261, 2002. 